Bull. Austral. Math. Soc.

VOL. 65 (2002) [153-174]

\title{
THE KUNZE-STEIN PHENOMENON ON THE ISOMETRY GROUP OF A TREE
}

\author{
Alessandro Veca
}

Let $G$ be the group of isometries of a homogeneous tree $\mathfrak{X}$. In the first part of this paper we decompose $G$ in terms of certain subgroups $N, \mathbb{Z}$ and $K$ to obtain the related integral formula

$$
\int_{G} f(z) d z=\int_{N} \sum_{j \in \mathbb{Z}} \int_{K} f\left(n \tau^{j} k\right) q^{-j} d k d \mu(n) .
$$

Then, by using ideas of $\mathrm{A}$. Ionescu and the formula above, we prove that

$$
L^{2,1}(G) * L^{2,1}(G) \subseteq L^{2, \infty}(G)
$$

and that a related maximal operator on $\mathfrak{X}$ is bounded from $L^{2,1}(\mathfrak{X})$ to $L^{2, \infty}(\mathfrak{X})$. We finally show that $L^{p, 1}(K \backslash G / K)$ is a commutative Banach algebra of convolutors for $L^{p}(G)$ and give an explicit description of its Gelfand spectrum.

\section{INTRODUCTION}

The principal aim of this paper is to prove that if $G$ is the group of isometries of a homogeneous tree $\mathfrak{X}$, then (Theorem 6.4)

$$
L^{2,1}(G) * L^{2,1}(G) \subseteq L^{2, \infty}(G) .
$$

The continuous inclusion (1.1) is called the generalised Kunze-Stein phenomenon for $G$. The classical Kunze-Stein phenomenon

$$
L^{2}(G) * L^{p}(G) \subseteq L^{2}(G) \quad 1 \leqslant p<2
$$

was shown for $G=S L(2, \mathbb{R})$ by Kunze and Stein [6] and for every semisimple Lie group with finite centre by Cowling [1]. Cowling, Meda and Setti stated a more accurate version of this phenomenon, by using the Lorentz spaces $L^{p, q}(G)$ (see [2] and [3]). Finally, in 2000 , Ionescu showed that the continuous inclusion (1.1) holds for every semisimple Lie group of real rank one and finite centre [5]. We present here a detailed version of his proof, adapted to the homogeneous tree case.

Received 20th August, 2001

I thank Michael Cowling and Stefano Meda for their helpful suggestions and useful hints.

Copyright Clearance Centre, Inc. Serial-fee code: 0004-9727/02 \$A2.00+0.00. 
The key ingredient in Ionescu's proof is the relation between Iwasawa and Cartan decompositions for semisimple Lie groups. In Section 3 we introduce and study in some detail a suitable subgroup $N$ (see [4]) which leads to a decomposition "of Iwasawa type" for the group $G$ of isometries of a homogeneous tree (Theorem 3.5). Although this decomposition is not a bijection, there is a related integral formula

$$
\int_{G} f(z) d z=\int_{N} \sum_{j \in \mathbf{Z}} \int_{K} f\left(n \tau^{j} k\right) q^{-j} d k d n
$$

where $f$ is a compactly supported continuous function on $G, \tau$ is a one step translation in $G, K$ is the isotropy group of a base point $o$ in $\mathfrak{X}$ and $d k$ and $d n$ are the Haar measures on $K$ and $N$ respectively. The analogy with the Iwasawa decomposition for Lie groups is apparent.

In Section 5 we show that the maximal operator

$$
\widetilde{M} f(x)=\sup _{r \in \mathbb{N}} \frac{1}{|B(x, r)|^{1 / 2}} \sum_{y \in B(x, r)}|f(y)|
$$

is bounded from $L^{2,1}(\mathfrak{X})$ to $L^{2, \infty}(\mathfrak{X})$ (following [5]) and, as a corollary, we obtain a $K$ bi-invariant version for the generalised Kunze-Stein phenomenon for $G$. In Section 6 we prove (1.1) and finally, in Section 7, we use the generalised Kunze-Stein phenomenon to show that there is a natural norm (see $[2,8,3]$ ) which makes $L^{p, 1}(K \backslash G / K)$ a commutative Banach algebra of convolutors of $L^{p}(G)$. We give an explicit description of the Gelfand spectrum of this subalgebra (Theorem 7.7).

\section{DEFINITIONS AND NOTATIONS}

A homogeneous tree of degree $q+1$ is a connected graph $\mathfrak{X}$ with no loops, in which every vertex is adjacent to $q+1$ other vertices. It carries a natural distance $d, d(x, y)$ being the number of edges between vertices $x$ and $y$. Let $S(x, r)$ and $B(x, r)$ denote the sphere and the closed ball centred at $x$ and of radius $r$ in the metric space $(\mathfrak{X}, d)$. Let II be a subset of $\mathbb{Z}$ of the form $I \cap \mathbb{Z}$, where $I$ is any (possibly infinite) interval of real numbers. A chain is a collection of vertices $\left\{x_{i}\right\}_{i \in \mathbb{I}}$ such that $d\left(x_{i}, x_{j}\right)=|i-j|$ whenever $i, j \in \mathbb{I}$. We say that a chain is a geodesic ray or a geodesic if $\mathbb{I}$ is equal to $\mathbb{N}$ or $\mathbb{Z}$ respectively. We say that a geodesic ray $\left\{x_{i}\right\}_{i \in \mathbf{N}}$ starts at $x$ if $x_{0}=x$. Geodesic rays $\left\{x_{i}\right\}_{i \in \mathbb{N}}$ and $\left\{y_{j}\right\}_{j \in \mathbb{N}}$ are identified if there exist integers $i$ and $j$ such that $x_{n}=y_{n+i}$ for every $n$ greater than $j$; this identification is an equivalence relation. We denote by $\Omega$ the set of equivalence classes and by $\Omega_{x}$ the set of all geodesic rays starting at $x$. Note that for every element in $\omega \in \Omega$ there exists a unique representative geodesic ray in $\Omega_{x}$ : we denote this geodesic ray by $[x, \omega)$.

Let $o$ and $\omega^{+}$be fixed but arbitrary reference points of $\mathfrak{X}$ and $\Omega$ respectively and assume that $\gamma^{+}=\left\{\gamma_{i}\right\}_{i \in \mathbf{N}}$ is the representative of $\omega^{+}$in $\Omega_{o}$. Define the height function $h$ 
(associated to $\omega^{+}$) by the rule

$$
h(x)=\lim _{i \rightarrow \infty}\left(i-d\left(x, \gamma_{i}\right)\right)
$$

For $j \in \mathbb{Z}$, we set

$$
\mathfrak{H}(j)=\{x \in \mathfrak{X}: h(x)=j\},
$$

so that $\mathfrak{X}$ decomposes disjointly: $\mathfrak{X}=\bigcup_{j \in \mathbb{Z}} \mathfrak{H}(j)$. The level sets $\mathfrak{H}(j)$ of the height function $h$ are called horocycles of $\mathfrak{X}$.

Denote by $G$ the group of isometries of $(\mathfrak{X}, d)$. The group $G$ acts transitively on $\mathfrak{X}$, is unimodular and locally compact, a neighbourhood basis being given by the sets of type

$$
U(g, E)=\{h \in G: h \cdot x=g \cdot x \quad \forall x \in E\},
$$

where $g$ is in $G$ and $E$ is a finite subset of $\mathfrak{X}$. It is easily satisfied that if $\left\{x_{i}\right\}_{i \in \mathbb{N}}$ and $\left\{y_{j}\right\}_{j \in \mathbb{N}}$ are equivalent geodesic rays, then $\left\{g \cdot x_{i}\right\}_{i \in \mathbb{N}}$ and $\left\{g \cdot y_{j}\right\}_{j \in \mathbb{N}}$ are equivalent geodesic rays. This defines a transitive action of $G$ on $\Omega$. We denote by $K_{x}$ and $G_{\omega}$ the stabilisers of $x \in \mathfrak{X}$ and $\omega \in \Omega$ under the $G$ action and write $K$ for $K_{o}$. Then $K$ is a maximal compact subgroup of $G$ and $\mathfrak{X}$ may be identified with the coset space $G / K$. A function $f$ on $\mathfrak{X}$ is said to be radial if it only depends on $d(x, o)$. We identify $K$-right-invariant and $K$-bi-invariant functions on $G$ with functions and radial functions on $\mathfrak{X}$ respectively.

We need some more notation. Given a topological space $X$, denote by $C_{c}(X)$ the set of continuous compactly supported functions on $X$.

If $f$ is a measurable function on a measure space $(X, \mu)$ define the distribution function $\lambda_{f}$ and nonincreasing rearrangement $f^{*}$ by

$$
\lambda_{f}(s)=\mu\{x \in X:|f(x)|>s\} \quad \text { and } f^{*}(t)=\inf _{s \in \mathbb{R}^{+}}\left\{s: \lambda_{f}(s) \leqslant t\right\} .
$$

Consider a measurable function $F: X \times X \mapsto \mathbb{R}^{+}$. Denote by $F^{* *}(\cdot, \cdot)$ the rearrangement of the function $x \mapsto F^{*}(x, \cdot)$, where $F^{*}(x, \cdot)$ is the rearrangement of $y \mapsto F(x, y)$. The function $F^{* *}$ is called the double rearrangement of $F$. We summarise some properties of rearrangements and double rearrangements in the next lemma.

Lemma 2.1. Let $f: X \mapsto \mathbb{R}^{+}$and $F: X \times X \mapsto \mathbb{R}^{+}$be measurable functions. Then

(i) $\left(\int_{X} f(x)^{2} d \mu(x)\right)^{1 / 2}=\left(\int_{0}^{\mu(X)} f^{*}(t)^{2} d t\right)^{1 / 2} ;$

(ii) $\int_{X} \int_{X} F(x, y) d \mu(x) d \mu(y)=\int_{0}^{\mu(X)} \int_{0}^{\mu(X)} F^{* *}(s, t) d s d t$;

(iii) if $D$ and $E$ are measurable subsets of $X$ of measures $\delta$ and $\varepsilon$, then

$$
\int_{D} \int_{E} F(x, y) d \mu(x) d \mu(y) \leqslant \int_{0}^{\delta} \int_{0}^{\varepsilon} F^{* *}(s, t) d s d t .
$$




\section{THE GROUP $N$ AND THE BOUNDARY $\Omega$}

Recall that $\gamma^{+}=\left\{\gamma_{i}\right\}_{i \in \mathbf{N}}$ is a reference geodesic ray in $\Omega_{o}$ and that $G_{\omega^{+}}$is the stabiliser of its equivalence class $\omega^{+}$. Choose another geodesic ray $\gamma^{-}=\left\{\gamma_{-i}\right\}_{i \in N}$ such that $\gamma^{-} \cap \gamma^{+}=\{o\}$ and write $\gamma$ for the geodesic $\left\{\gamma_{i}\right\}_{i \in \mathbf{Z}}$. Note that $h\left(\gamma_{j}\right)=j$ for all $j \in \mathbb{Z}$. Given $\omega_{1}$ and $\omega_{2}$ in $\Omega$ there exists a unique (up to renumbering) geodesic $\left\{y_{i}\right\}_{i \in \mathbb{Z}}$ such that $\omega_{1}$ and $\omega_{2}$ are the equivalence classes of $\left\{y_{i}\right\}_{i \in \mathbf{N}}$ and $\left\{y_{-i}\right\}_{i \in \mathbb{N}}$ respectively. For brevity, we denote this geodesic by $\left(\omega_{1}, \omega_{2}\right)$ disregarding the labels.

Let $N$ be the subset of all elements in $G_{\omega^{+}}$which also fix some vertex of $\mathfrak{X}$ (see [4]). The next lemma gives a description of $N$ in terms of the height function $h$.

LEMma 3.1. An isometry $z$ belongs to $N$ if and only if $h(z \cdot x)=h(x)$ for every $x \in \mathfrak{X}$. Further $N$ is a closed subgroup of $G$.

Proof: Let $n$ be in $N$. By definition $n \cdot \omega^{+}=\omega^{+}$and $n \cdot x=x$ for some $x \in \mathfrak{X}$, so that $n$ fixes every vertex in the geodesic ray $\left[x, \omega^{+}\right)$starting at $x$ and equivalent to $\omega^{+}$. It follows that $N$ is the set of those elements in $G$ that fix the elements $\gamma_{i}$ of $\gamma^{+}$for $i$ big enough and it is therefore apparent that $N$ is a subgroup. In particular $n^{-1}$ fixes the elements in $\gamma^{+}$eventually and

$$
h(n \cdot x)=\lim _{j \rightarrow \infty}\left(j-d\left(x, n^{-1} \cdot \gamma_{j}\right)\right)=\lim _{j \rightarrow \infty}\left(j-d\left(x, \gamma_{j}\right)\right)=h(x)
$$

On the other hand, suppose $z \in G$ satisfies $h(z \cdot y)=h(y)$ for every $y \in \mathfrak{X}$. Let $x=z \cdot o$, assume $\left[x, \omega^{+}\right)=\left\{x_{i}\right\}_{i \in \mathbb{N}}$ and note that $x_{i}=\gamma_{i}$ for sufficiently large $i$. Observe also that, for any $j$ in $\mathbb{N}, d\left(z \cdot \gamma_{j}, x\right)=j$ and $h\left(z \cdot \gamma_{j}\right)=h\left(\gamma_{j}\right)=j$. Since $S(x, j) \cap \mathfrak{H}(j)$ reduces to the point $\left\{x_{j}\right\}$, we conclude that $z \cdot \gamma_{j}=x_{j}$ and then $z$ belongs to $N$. We finally show that $N$ is closed by proving that its complement $N^{c}$ is open in $G$. Let $z$ be in $N^{c}$. Then there exists $x \in \mathfrak{X}$ such that $h(z \cdot x) \neq h(x)$, so that the set

$$
U(z,\{x\})=\{g \in G: g \cdot x=z \cdot x\}
$$

is an open neighbourhood of $z$, contained in $N^{c}$.

CoRollary 3.2. The orbit of $x \in \mathfrak{X}$ under the $N$ action is exactly the horocycle $\mathfrak{H}(h(x))$.

Proof: Choose $y \neq x$ in $\mathfrak{H}(h(x))$ and a vertex $\gamma_{i}$ in

$$
\gamma \cap\left[x, \omega^{+}\right) \cap\left[y, \omega^{+}\right) .
$$

It is clear that $i>h(x)$ and that $x, y \in S\left(\gamma_{i}, i-h(x)\right)$. Since elements of $K_{\gamma_{i}}$ act as permutations on $S\left(\gamma_{i}, i-h(x)\right)$ (and every permutation of $S\left(\gamma_{i}, i-h(x)\right)$ derives from an element of $K_{\gamma_{1}}$ ) there exists (actually infinitely many) $n \in G_{\omega^{+}} \cap K_{\gamma_{i}} \subset N$ such that. $n \cdot x=y$ and the claim is proved. 
We denote by $N_{x}$ the stabiliser of $x \in \mathfrak{X}$ under the $N$ action and we write $N_{i}$ for $N_{\gamma_{i}}$ for all $i \in \mathbb{Z}$. The following lemma describes some properties of the Haar measure $\mu$ on the locally compact group $N$. If $E$ is a finite set, then we write $|E|$ for the number of its elements.

Lemma 3.3. Let $\mu, N_{x}$ and $h$ be as above. Then

(i) the measure $\mu$ can be normalised such that $\mu\left(N_{x}\right)=q^{h(x)}$ for all $x \in \mathfrak{X}$;

(ii) $N$ is unimodular.

REMARK 3.4. In the following we shall assume that the Haar measure on $N$ is normalised as in (i).

Proof: To prove part (i), first note that $N_{x}=K_{x} \cap N$ is a compact subgroup of $N$ and so its measure in $N$ is finite. Moreover, since $\mathfrak{X}$ is countable and $N=\bigcup_{x \in \mathfrak{X}} N_{x}$, there exists $\widetilde{x} \in \mathfrak{X}$ such that $\mu\left(N_{\tilde{x}}\right)>0$. Let $y$ be the unique vertex such that $d(\widetilde{x}, y)=1$ and $h(y)=h(\widetilde{x})+1$. It is clear that $N_{y}$ acts transitively on $S(y, 1) \cap \mathfrak{H}(h(\widetilde{x}))$ and that the stabiliser of $\widetilde{x}$ in $N_{y}$ is exactly $N_{\tilde{x}}$. From the left invariance of $\mu$ and since the index of $N_{\tilde{x}}$ in $N_{y}$ is equal to $|S(y, 1) \cap \mathfrak{H}(h(\widetilde{x}))|=q$, it follows that

$$
\mu\left(N_{\mathfrak{y}}\right)=\mu\left(\bigcup_{i=1}^{q} z_{i} N_{\tilde{x}}\right)=\sum_{i=1}^{q} \mu\left(z_{i} N_{\tilde{x}}\right)=q \mu\left(N_{\tilde{x}}\right),
$$

$\left\{z_{i}\right\}_{i=1, \ldots, q}$ being a complete family of representatives for the coset space $N_{y} / N_{\tilde{x}}$. With a similar argument it turns out that $\mu\left(N_{x}\right)=q^{h(x)-h(\tilde{x})}$ for all $x \in \mathfrak{X}$ and, normalising $\mu$ so that $\mu\left(N_{o}\right)=1$, we have $\mu\left(N_{x}\right)=q^{h(x)}$ as required.

To show part (ii), let $n$ be an element in $N$ and let $\Delta_{N}$ denote the modular function on $N$ (that is, the function such that $\mu(A n)=\Delta_{N}(n) \mu(A)$ for any measurable set $A$ ). Using part (i) and the fact $n^{-1} N_{x} n=N_{n^{-1} \cdot x}$ we have

$$
\mu\left(N_{x}\right)=q^{h(x)}=q^{h\left(n^{-1} \cdot x\right)}=\mu\left(N_{n^{-1} \cdot x}\right)=\mu\left(n^{-1} N_{x} n\right)=\mu\left(N_{x} n\right)=\Delta_{N}(n) \mu\left(N_{x}\right),
$$

which implies $\Delta_{N}=1$.

The main result of this section is contained in Theorem 3.5 below which underlines the analogies between $G$ and semisimple Lie groups of rank one. Let $\tau$ be a fixed one-step translation along the reference geodesic $\gamma$ towards $\omega^{+}$, that is, $\tau \cdot \gamma_{j}=\gamma_{j+1}$ for all $j \in \mathbb{Z}$.

TheOREM 3.5. Let $G, N, K$ and $\tau$ be as above. Then for every $z \in G$ there exist $n \in N, j \in \mathbb{Z}$ and $k \in K$ such that $z=n \tau^{j} k$. Further, if $f$ is a continuous compactly supported function on $G$, then

$$
\int_{G} f(z) d z=\int_{N} \sum_{j \in \mathbb{Z}} \int_{K} f\left(n \tau^{j} k\right) q^{-j} d k d \mu(n) .
$$


Proof: An element in $G$ must be a translation along some geodesic, a rotation around some vertex or an inversion (an isometry which exchanges two adjacent vertices) (see [4], Chapter 2). Since an inversion can not fix any point in $\Omega$, an element $z_{\omega^{+}}$ of $G_{\omega^{+}} \backslash N$ is a translation along some geodesic $\left\{x_{i}\right\}_{i \in \mathbf{Z}}$ with $\left\{x_{i}\right\}_{i \in \mathbf{N}}$ equivalent to $\gamma^{+}$. Choose an element $x$ lying on both $\gamma$ and $\left\{x_{i}\right\}_{i \in \mathbf{Z}}$ and let $j_{0}$ be the integer such that $z_{\omega^{+}} \cdot x_{j}=x_{j+j_{0}}$ for all $j \in \mathbb{Z}$. It is clear that $z_{\omega^{+}} \tau^{-j}$ fixes $\omega^{+}$and $x$ so that $z_{\omega^{+}} \tau^{-j_{0}} \in N$. It follows that each element in $G_{\omega^{+}}$may be written as the product of an element of $N$ and an element of the subgroup generated by $\tau$. Finally, if $z$ is a generic element of $G$, there exists $k \in K$ such that $k \cdot \omega^{+}=z \cdot \omega^{+}$so that $z k^{-1}$ belongs to $G_{\omega^{+}}$, and there exist $n \in N$ and $j \in \mathbb{Z}$ such that $z k^{-j}=n \tau^{j}$, as required. To show the second part of the theorem, suppose first $F$ is a $K$-invariant function on $G$. We can write

$$
\int_{G} F(z) d z=\sum_{x \in \mathfrak{X}} F(x)=\sum_{j \in \mathbf{Z}} \sum_{x \in \mathfrak{S}(j)} F(x) .
$$

We know that $N$ acts transitively on $\mathfrak{H}(j)$ for all $j \in \mathbb{Z}$. Denote by $\{n(x)\}_{x \in \mathfrak{H}(j)}$ a complete family of representatives for the cosets of $N_{j}$ in $N$. We have that $N=\bigcup_{x \in \mathfrak{H}(j)} n(x) N_{j}$ (disjoint union) and that $\mu\left(n(x) N_{j}\right)=\mu\left(N_{j}\right)=q^{j}$ in view of Lemma 3.3. We have

$$
\begin{aligned}
\sum_{x \in \mathfrak{S}(j)} F(x) & =\sum_{x \in \mathfrak{H}(j)}\left(\frac{1}{\mu\left(n(x) N_{j}\right)} \int_{n(x) N_{j}} d \mu\right) F(x) \\
& =\sum_{x \in \mathfrak{H}(j)} q^{-j} \int_{n(x) N_{j}} F\left(n \tau^{j} \cdot o\right) d n \\
& =q^{-j} \int_{N} F\left(n \tau^{j} \cdot o\right) d n,
\end{aligned}
$$

and then, by (3.3),

$$
\int_{G} F(z) d z=\sum_{j \in \mathbf{Z}} q^{-j} \int_{N} F\left(n \tau^{j} \cdot o\right) d n
$$

If $f \in C_{c}(G)$, then $F(z)=\int_{K} f(z k) d k$ is $K$-invariant and $\int_{G} f(z) d z=\int_{G} F(z) d z$. The desired result follows from (3.4).

REMARK 3.6. Observe that the decomposition in Theorem 3.5 is not unique. Denote by $\pi$ the projection $(n, j, k) \mapsto n \tau^{j} k$ and suppose $z=\pi(n, j, k)=\pi\left(n_{1}, j_{1}, k_{1}\right)$. It follows that $j=j_{1}$ because

$$
j=h\left(\gamma_{j}\right)=h\left(n \cdot \gamma_{j}\right)=h\left(n \tau^{j} k \cdot o\right)=h\left(n_{1} \tau^{j_{1}} k_{1} \cdot o\right)=j_{1} .
$$

Furthermore $k^{-1} \omega^{+}=z^{-1} \cdot \omega^{+}=k_{1}^{-1} \omega^{+}$and then $k k_{1}^{-1} \in G_{\omega^{+}} \cap K=N_{o}$. There exists $n_{o} \in N_{o}$ such that $k=n_{o} k_{1}$ so that $n \tau^{j} k=n\left(t^{j} n_{o} \tau^{-j}\right) \tau^{j} k_{1}$. It follows that $n_{1}=$ $n\left(t^{j} n_{\mathrm{o}} \tau^{-j}\right)$ and that $\pi$ is constant on the sets of $N \times \mathbb{Z} \times K$ of type $\left\{\left(n \tau^{j} n_{o} \tau^{-j}, j, n_{o}^{-1} k\right)\right.$ : $\left.n_{o} \in N_{o}\right\}$. 
Remark 3.7. Consider a function $f$ on $\mathfrak{X}$ (that is, a $K$-right-invariant function on $G$ ). In this case it may be useful to rewrite formula (3.2) as an integral on $\Omega$ instead of as $N$. Note that for any $\omega$ in $\Omega \backslash\left\{\omega^{+}\right\}$, the geodesic $\left(\omega, \omega^{+}\right)$intersects $\mathfrak{H}(j)$ in exactly one point, so that the map $\Phi: \Omega \times \mathbb{Z} \rightarrow \mathfrak{X}$, defined by the rule $\Phi(\omega, j)=\mathfrak{H}(j) \cap\left(\omega, \omega^{+}\right)$, is well defined. This map is surjective and $\Phi^{-1}(x)=\Omega(x) \times\{h(x)\}$, where $\Omega(x)=\{\omega \epsilon$ $\left.\Omega: x \in\left(\omega, \omega^{+}\right)\right\}$. Set $\rho\left(\left\{\omega^{+}\right\}\right)=0$ and $\rho(\Omega(x))=q^{h(x)}$, so that $\rho$ extends to a Borel measure on $\Omega$. Since $\omega \in \Omega(x)$ if and only if $x \in\left(\omega, \omega^{+}\right)$, given an integrable function on $\mathfrak{X}$ we have

$$
\begin{aligned}
\sum_{x \in \mathcal{X}} f(x) & =\sum_{x \in \mathcal{X}} \rho(\Omega(x))^{-1} \int_{\Omega(x)} d \rho(\omega) f(x) \\
& =\int_{\Omega} \sum_{x \in\left(\omega, \omega^{+}\right)} q^{-h(x)} f(x) d \rho(\omega) \\
& =\int_{\Omega} \sum_{j \in \mathbf{Z}} q^{-j} f(\Phi(\omega, j)) d \rho(\omega) .
\end{aligned}
$$

LEMma 3.8. Let $f$ be in $C_{c}(N)$. The map $n \mapsto \tau^{-j} n \tau^{j}$ is an automorphism of $N$ and

$$
\int_{N} f\left(\tau^{-j} n \tau^{j}\right) d n=q^{j} \int_{N} f(n) d n
$$

Proof: Let $j$ be an integer. Since

$$
h\left(\tau^{-j} n \tau^{j} \cdot x\right)=h\left(n \tau^{j} \cdot x\right)-j=h\left(\tau^{j} \cdot x\right)-j=h(x),
$$

we have $\tau^{-\jmath} n \tau^{j} \in N$ in view of Lemma 3.1, so that $n \mapsto \tau^{-j} n \tau^{j}$ is an automorphism. Let $\Delta$ be the modular function associated to this automorphism, that is, the group homomorphism $\Delta: \mathbb{Z} \rightarrow \mathbb{R}^{+}$such that

$$
\int_{N} f\left(\tau^{-j} n \tau^{j}\right) d n=\Delta(j) \int_{N} f(n) d n
$$

To show that $\Delta(j)=q^{j}$ for all integers $j$, consider $f \in C_{c}(G / K)$. In view of Theorem 3.5 and since

$$
\mu\left\{n: n \cdot \gamma_{j}=x\right\}=q^{j} \quad \forall j \in \mathbb{Z}, \forall x \in \mathfrak{H}(j),
$$

we have

$$
\begin{aligned}
\sum_{x \in \mathcal{X}} f(x) & =\sum_{i \in \mathbf{Z}} \int_{N} f\left(n \tau^{i} \cdot o\right) q^{-i} d n \\
& =\Delta(j)^{-1} \sum_{i \in \mathbb{Z}} \int_{N} f\left(\tau^{-j} n \tau^{j+i} \cdot o\right) q^{-i} d n \\
& =\Delta(j)^{-1} \sum_{i \in \mathbf{Z}} \sum_{x \in \mathfrak{S}(j+i)} \int_{\left\{n: n \cdot \gamma_{j+i}=x\right\}} f\left(\tau^{-j} n \cdot \gamma_{j+i}\right) q^{-i} d n
\end{aligned}
$$




$$
\begin{aligned}
& =\Delta(j)^{-1} \sum_{i \in \mathbf{Z}} \sum_{x \in \mathcal{S}(j+i)} f\left(\tau^{-j} \cdot x\right) q^{j} \\
& =\Delta(j)^{-1} q^{j} \sum_{x \in \mathcal{X}} f(x)
\end{aligned}
$$

which implies $\Delta(j)=q^{j}$. The desired result now follows from (3.6).

Recall that $\mathfrak{X}=\bigcup_{j \in \mathbf{Z}} \mathfrak{H}(j)$ and note that $\mathfrak{X}=\bigcup_{r \in \mathbb{N}} S(o, r)$. The first decomposition for $\mathfrak{X}$ is associated to the decomposition for $G$ described in Theorem 3.5. The second one is related to the Cartan decomposition $G=\bigcup_{r \in \mathbb{N}} K \tau^{r} K$. Lemmas 3.10 and 3.11 below relate these decompositions.

Let

$$
\mathfrak{B}(x, r)=B(x, r) \cap \mathfrak{H}(h(x))=\{y \in B(x, r): h(x)=h(y)\} .
$$

REMARK 3.9. Note that the intersection of two such "balls" $\mathfrak{B}(x, r)$ and $\mathfrak{B}\left(x^{\prime}, r^{\prime}\right)$ is always trivial. It is simple to see that if $\mathfrak{B}(x, r) \cap \mathfrak{B}\left(x^{\prime}, r^{\prime}\right) \neq \emptyset$, then $\mathfrak{B}(x, r) \subseteq \mathfrak{B}\left(x^{\prime}, r^{\prime}\right)$ or $\mathfrak{B}\left(x^{\prime}, r^{\prime}\right) \subseteq \mathfrak{B}(x, r)$.

If $\alpha$ is a nonnegative real number, then we write $\lfloor\alpha\rfloor$ for the maximum $n \in \mathbb{N}$ such that $n \leqslant \alpha$.

LEMMA 3.10. Let $x \in \mathfrak{X}, \omega \in \Omega(x), r \in \mathbb{N}$ and let $h$ be an integer such that $r \geqslant|h|$. Then

(i) $B(x, r) \cap \mathfrak{H}(h(x)+h)=\mathfrak{B}(\Phi(\omega, h(x)+h), 2\lfloor(r-h) / 2\rfloor)$

(ii) $|B(x, r) \cap \mathfrak{H}(h(x)+h)|=q^{\lfloor(r-h) / 2\rfloor}$.

Proof: Observe that the result does not depend on the particular choice of $\omega \in$ $\Omega(x)$ because of the intersection properties of the balls $\mathfrak{B}$. Suppose first that $r-h$ is even. It is not too hard to see that to reach $\mathfrak{H}(h(x)+h)$ in exactly $r$ steps starting from $x$, it is necessary to move toward $\omega^{+}$along the infinite chain $\left[x, \omega^{+}\right)$by $(r+h) / 2$ steps, first arriving at the point $y=\Phi(\omega, h(x)+(r+h) / 2)$ and then going down by $(r-h) / 2$ steps along $\left[y, \omega^{\prime}\right), \omega^{\prime}$ being an arbitrary point in $\Omega(y)$. This implies that

$$
B(x, r) \cap \mathfrak{H}(h(x)+h)=S(y,(r-h) / 2) \cap \mathfrak{H}(h(x)+h)=\mathfrak{B}(\Phi(\omega, h(x)+h), r-h) .
$$

If $r-h$ is odd, a similar argument shows that if $y^{\prime}=\Phi(\omega, h(x)+(r+h-1) / 2)$ then

$B(x, r) \cap \mathfrak{H}(h(x)+h)=S\left(y^{\prime},(r-h-1) / 2\right) \cap \mathfrak{H}(h(x)+h)=\mathfrak{B}(\Phi(\omega, h(x)+h), r-h-1)$

and part (i) is proved; since finally $|\mathfrak{B}(x, r)|=q^{\lfloor r / 2\rfloor}$ for any $x \in \mathfrak{X}$ and $r \in \mathbb{N}$, part (ii) easily follows from part (i).

Let $j$ be an integer and $r \in \mathbb{N}$ and set

$$
T_{r, j}=\left\{n \in N: n \cdot \gamma_{j} \in S(o, r)\right\}
$$

and $\psi(r, j)=q^{-j / 2} \mu\left(T_{r, j}\right)$. It is clear that $T_{r, j}=\emptyset$, and then $\psi(r, j)=0$, if $r<|j|$. 
Lemma 3.11. Suppose $j \in \mathbb{Z}, r \in \mathbb{N}$ and $r \geqslant|j|$. Then $T_{r, j}$ is an open subset of $N$ and

$$
\mu\left(T_{r, j}\right)= \begin{cases}0 & \text { if } r-j \text { is odd } \\ q^{j} & \text { if } j=r \\ 1 & \text { if } j=-r \\ \frac{q-1}{q} q^{(r+j) / 2} & \text { otherwise. }\end{cases}
$$

Consequently $\psi(r, j) \leqslant q^{r / 2}$ for every $(r, j) \in \mathbb{N} \times \mathbb{Z}$.

Proof: By using the same circle of ideas as Lemma 3.10 it is easy to see that

$$
b(r, j)=|S(o, r) \cap \mathfrak{H}(j)|= \begin{cases}0 & \text { if } r-j \text { is odd } \\ 1 & \text { if } j=r \\ q^{-j} & \text { if } j=-r \\ \frac{q-1}{q} q^{(r-j) / 2} & \text { otherwise. }\end{cases}
$$

Let $\left\{y_{i}\right\}_{i=1, \ldots, b(r, j)}$ be the elements of $S(o, r) \cap \mathfrak{H}(j)$. Then $T_{r, j}$ is the disjoint union of $\left\{n: n \cdot \gamma_{j}=y_{i}\right\}$ where the index $i$ varies over $1, \ldots, b(r, j)$. We conclude in particular that $T_{r, j}$ is open and that

$$
\mu\left(T_{r, j}\right)=\sum_{i=1}^{b(r, j)} \mu\left\{n: n \cdot \gamma_{j}=y_{i}\right\}=b(r, j) \mu\left(N_{j}\right)=q^{j} b(r, j)
$$

as required, by the left invariance of $\mu$.

\section{THE ROLE OF $L^{2,1}$}

In this section we present some simple results which make clear the role played by $L^{2,1}$ in the following sections. We say that a measurable function $f$ on a measure space $X$ belongs to the Lorentz space $L^{p, q}(X)$ if and only if $\|f\|_{p, q}<\infty$, where

$$
\|f\|_{p, q}= \begin{cases}\left(\frac{q}{p} \int_{0}^{\infty}\left(t^{1 / p} f^{*}(t)\right)^{q} \frac{d t}{t}\right)^{1 / q} & 1<p<\infty, q<\infty \\ \sup _{t>0}\left(t^{1 / p} f^{*}(t)\right)=\sup _{s>0}\left(s^{p} \lambda_{f}(s)\right)^{1 / p} & 1<p \leqslant \infty, q=\infty .\end{cases}
$$

For more details about Lorentz spaces, see [10]. Note that the lemmas below depend crucially on the following property of the quasinorm $\|\cdot\|_{p, q}$ : let $E$ be a measurable subset of a measure space $(X, \mu)$, then $\left\|\chi_{E}\right\|_{p, q}=\mu(E)^{1 / p}$ for any $p \in(1, \infty)$ and $q \in[1, \infty]$.

Let $f$ be a finitely supported function on $\mathfrak{X}$ and let

$$
\mathfrak{M} f(x)=\sup _{r \in \mathbb{N}} \frac{1}{|\mathfrak{B}(x, r)|} \sum_{y \in \mathfrak{B}(x, r)}|f(y)| .
$$


Proposition 4.1. The operator $\mathfrak{M}$ is of weak type $(1,1)$ and of type $(p, p)$ for any $p>1$. Consequently $\mathfrak{M}$ is bounded from $L^{2,1}(G / K)$ to itself.

PROOF: Let $f$ be a finitely supported function on $\mathfrak{X}$ and set

$$
F_{s}=\{y \in \mathfrak{X}:|\mathfrak{M} f(y)|>s\} .
$$

If $y \in F_{s}$, then there exists $r_{y}$ such that

$$
\frac{1}{\left|\mathfrak{B}\left(y, r_{y}\right)\right|} \sum_{z \in \mathfrak{B}\left(y, r_{y}\right)}|f(z)|>s .
$$

Since $\mathfrak{B}\left(y, r_{y}\right)=\mathfrak{B}\left(w, r_{y}\right)$ if $w \in \mathfrak{B}\left(y, r_{y}\right)$, it follows that

$$
\mathfrak{B}\left(y, r_{y}\right) \subset F_{s} .
$$

It is possible to find $x_{1}, \ldots, x_{l}$ such that $F_{s}$ decomposes disjointly as $\bigcup_{i=1}^{l} \mathfrak{B}\left(x_{i}, r_{x_{i}}\right)$ so that

$$
\left|F_{s}\right|=\sum_{i=1}^{l}\left|\mathfrak{B}\left(x_{i}, r_{x_{i}}\right)\right| \leqslant \frac{1}{s} \sum_{i=1}^{l} \sum_{y \in \mathfrak{B}\left(x_{i}, r_{x_{i}}\right)}|f(y)|=\sum_{y \in F_{s}}|f(y)| \leqslant \frac{\|f\|_{1}}{s}
$$

hence $\mathfrak{M}$ is of weak type $(1,1)$. The $L^{p}$ and $L^{2,1}$ boundedness follow from the trivial $L^{\infty}$ boundedness and from interpolation theorems.

LEMmA 4.2. Let $X_{1}$ and $X_{2}$ be measurable spaces with measure $\mu_{1}$ and $\mu_{2}$. Then

$$
\left(\int_{X_{1}}\|F(x, \cdot)\|_{L^{2,1}\left(X_{2}\right)}^{2} d \mu_{1}(x)\right)^{1 / 2} \leqslant C\|F\|_{L^{2,1}\left(X_{1} \times X_{2}\right)} \quad \forall F \in L^{2,1}\left(X_{1} \times X_{2}\right) .
$$

PROOF: It is sufficient to assume that $F=\chi_{E}$ is a characteristic function of a set $E$ of finite measure. We have

$\int_{X_{1}}\|F(x, \cdot)\|_{L^{2,1}\left(X_{2}\right)}^{2} d \mu_{1}(x)=\int_{X_{1}}\|F(x, \cdot)\|_{L^{2}\left(X_{2}\right)}^{2} d \mu_{1}(x)=\|F\|_{L^{2}\left(X_{1} \times X_{2}\right)}^{2}=\|F\|_{L^{2,1}\left(X_{1} \times X_{2}\right)}^{2}$, so that the result is proved.

LEMMA 4.3. Suppose $\beta$ is a nonzero real number and let $\nu_{1}$ and $\nu_{2}$ be the measures on $\mathbb{Z}$ such that $\nu_{1}(j)=\mathrm{e}^{\beta j}$ and $\nu_{2}(j)=\mathrm{e}^{2 \beta j}$. Then

$$
\|f\|_{L^{1}\left(\mathbf{Z}, \nu_{1}\right)} \leqslant\|f\|_{L^{2,1}\left(\mathbf{Z}, \nu_{2}\right)} \quad \forall f \in L^{2,1}\left(\mathbb{Z}, \nu_{2}\right) .
$$

Proof: Suppose $\beta<0$. Let $E$ be a finite subset of $\mathbb{Z}$, so that $j_{0}=\min (E)$ is well defined, and $f=\chi_{E}$. We have

$$
\|f\|_{L^{1}\left(\mathbb{Z}, \nu_{1}\right)}=\sum_{j \in E} \mathrm{e}^{\beta j} \leqslant \sum_{j \geqslant j_{0}} \mathrm{e}^{\beta j}=C_{\beta} \mathrm{e}^{\beta j 0} \leqslant C_{\beta}\left(\sum_{j \in E} \mathrm{e}^{2 \beta j}\right)^{1 / 2}=C_{\beta}\|f\|_{L^{2,1}\left(\mathbb{Z}, \nu_{2}\right)} .
$$

If $\beta>0$ the proof is similar. 


\section{A MAXIMAL OPERATOR ON $\mathfrak{X}$}

The purpose of this section is to show that the operator

$$
\widetilde{M} f(x)=\sup _{r \in \mathbb{N}} \frac{1}{|B(x, r)|^{1 / 2}} \sum_{y \in B(x, r)}|f(y)|
$$

is bounded from $L^{2,1}(G / K)$ to $L^{2, \infty}(G / K)$. We use the same ideas as Ionescu [5], who showed an analogous result in the case of noncompact Lie groups of real rank one.

THEOREM 5.1. The operator $\widetilde{M}$ is bounded from $L^{2,1}(G / K)$ to $L^{2, \infty}(G / K)$.

Proof: Observe first that

$$
\sum_{y \in B(x, r)}|f(y)|=\sum_{h \in \mathbf{Z}} \sum_{\substack{y \in S(h(x)+h) \\ y \in B(x, r)}}|f(y)| .
$$

Write $\mathfrak{B}(x, r, h)$ for $B(x, r) \cap \mathfrak{H}(h(x)+h)$ and choose $\omega$ in $\Omega(x)$. We may assume that $r \geqslant|h|$, otherwise $\mathfrak{B}(x, h, r)=\emptyset$. In view of part (ii) of Lemma 3.10,

$$
|\mathfrak{B}(x, r, h)|=q^{\lfloor(r-h) / 2\rfloor} \leqslant q^{(r-h) / 2}
$$

and

$$
\sum_{\substack{y \in \mathcal{S}(h(x)+h) \\ y \in B(x, r)}}|f(y)|=\sum_{y \in \mathfrak{B}(x, r, h)}|f(y)| \leqslant q^{(r-h) / 2} \mathfrak{M} f(\Phi(\omega, h(x)+h))
$$

so that

$$
\begin{aligned}
\widetilde{M} f(x)=\widetilde{M} f(\Phi(\omega, h(x))) & \leqslant C \sup _{r \in \mathbb{N}} q^{-r / 2} \sum_{y \in B(x, r)}|f(y)| \\
& \leqslant C \sum_{h \in \mathbf{Z}} q^{-h / 2} \mathfrak{M} f(\Phi(\omega, h(x)+h)) \\
& =C \sum_{j \in \mathbf{Z}} q^{(h(\boldsymbol{x})-j) / 2} \mathfrak{M} f(\Phi(\omega, j)) .
\end{aligned}
$$

In view of formula (3.5), the measure in $\mathfrak{X}$ of $E_{\lambda}=\{x \in \mathfrak{X}: \widetilde{M} f(x)>\lambda\}$ is equal to the measure of $\Phi^{-1}\left(E_{\lambda}\right)$ in $\Omega \times \mathbb{Z}$, and from (5.9),

$$
\begin{aligned}
\Phi^{-1}\left(E_{\lambda}\right) & \subseteq\left\{(\omega, l) \in \Omega \times \mathbb{Z}: \sum_{j \in \mathbb{Z}} q^{(l-j) / 2} \mathfrak{M} f(\Phi(\omega, j))>\lambda\right\} \\
& =\left\{(\omega, l) \in \Omega \times \mathbb{Z}: q^{l / 2} \sum_{j \in \mathbb{Z}} q^{-j / 2} \mathfrak{M} f(\Phi(\omega, j))>\lambda\right\} \\
& =\left\{(\omega, l) \in \Omega \times \mathbb{Z}: l / 2>\log _{q} \frac{\lambda}{\sum_{j} q^{-j / 2} \mathfrak{M} f(\Phi(\omega, j))}\right\}
\end{aligned}
$$


It follows that, if $g(\omega)=\log _{q} \lambda^{2}\left(\sum_{j} q^{-j / 2} \mathfrak{M} f(\Phi(\omega, j))\right)^{-2}$ and $\left|E_{\lambda}\right|$ denotes the measure of $E_{\lambda}$ in $\mathfrak{X}$, then

$$
\begin{aligned}
\left|E_{\lambda}\right| & =\int_{\Omega} \sum_{l \in \mathbf{Z}} q^{-l} \chi_{\Phi^{-1}\left(E_{\lambda}\right)}(\omega, l) d \rho(\omega) \\
& \leqslant \int_{\Omega}\left(\sum_{l>g(\omega)} q^{-l}\right) d \rho(\omega) \\
& \leqslant C \lambda^{-2} \int_{\Omega}\left(\sum_{j \in \mathbf{Z}} q^{-j / 2} \mathfrak{M}(f(\Phi(\omega, j)))^{2} d \rho(\omega) .\right.
\end{aligned}
$$

Let $\beta=-1 / 2$ and denote by $\nu_{2}$ the measure on $\mathbb{Z}$ associated to the function $j \mapsto q^{2 \beta j}$ as in Lemma 4.3. By using Lemmas 4.2 and 4.3 ,

$$
\begin{aligned}
\|\widetilde{M} f\|_{2, \infty}^{2}=\sup _{\lambda>0} \lambda^{2}\left|E_{\lambda}\right| & \leqslant C \int_{\Omega}\left(\sum_{j \in \mathbf{Z}} q^{-j / 2} \mathfrak{M}(f)(\Phi(\omega, j))\right)^{2} d \rho(\omega) \\
& \leqslant C \int_{\Omega}\|\mathfrak{M}(f)(\cdot, \omega)\|_{L^{2,1}\left(\mathbf{Z}, \nu_{2}\right)}^{2} d \rho(\omega) \\
& \leqslant C\|\mathfrak{M}(f)\|_{L^{2,1}\left(\Omega \times \mathbf{Z}, \rho \times \nu_{2}\right)}^{2} \\
& =C\|\mathfrak{M}(f)\|_{L^{2,1}(x)}^{2}
\end{aligned}
$$

and the theorem follows from Proposition 4.1.

Observe that Theorem 5.1 implies a $K$-invariant version of the generalised KunzeStein phenomenon.

Corollary 5.2. Let $G$ denote the group of isometries of a homogeneous tree $\mathfrak{X}$ of degree $q+1$. Then the following continuous inclusion holds:

$$
L^{2,1}(G / K) * L^{2,1}(K \backslash G / K) \subseteq L^{2, \infty}(G / K)
$$

Proof: Let $f$ and $\phi$ be the characteristic functions of finite subsets of $\mathfrak{X}$ and suppose $\phi$ is radial. Let $M$ be $\max _{x \in \mathfrak{X}}\{|x|: \phi(x) \neq 0\}$ and observe that $\chi_{S(o, M)} \leqslant \phi \leqslant \chi_{B(o, M)}$ so that there exist two positive constants $c_{1}$ and $c_{2}$ such that

$$
c_{1} q^{M / 2} \leqslant\|\phi\|_{2,1} \leqslant c_{2} q^{M / 2}
$$


It follows that

$$
\begin{aligned}
f * \phi(x)=\int_{G} f(z \cdot o) \phi\left(z^{-1} g \cdot o\right) d z & =\int_{G} f(z \cdot o) \phi\left(g^{-1} z \cdot o\right) d z \\
& =\sum_{y \in \mathfrak{X}} f(y) \phi\left(g^{-1} \cdot y\right) \\
& =\sum_{n \in \mathbb{N}} \phi(n) \sum_{y: d(x, y)=n} f(y) \\
& \leqslant \sum_{y \in B(x, M)} f(y) \\
& \leqslant q^{M / 2} \widetilde{M} f(x)
\end{aligned}
$$

and then

$$
\|f * \phi\|_{2, \infty} \leqslant q^{M / 2}\|\widetilde{M} f\|_{2, \infty} \leqslant C q^{M / 2}\|f\|_{2,1} \leqslant C\|\phi\|_{2,1}\|f\|_{2,1}
$$

\section{AN ENDPOINT ESTIMATE FoR the GENERALISED KUNZE-STEIN PHENOMENON}

The main result of this section is to remove the $K$-invariance hypothesis in Corollary 5.2 , by showing the continuous inclusion

$$
L^{2,1}(G) * L^{2,1}(G) \subseteq L^{2, \infty}(G)
$$

An analogous statement in the case of real rank one noncompact Lie groups was proved by Ionescu [5] and we present here a detailed arboreal version of his proof. Observe first that to show (6.11) it is sufficient to prove that

$$
\int_{G} \int_{G} f(z) g\left(z^{-1} z^{\prime}\right) h\left(z^{\prime}\right) d z d z^{\prime} \leqslant C\|f\|_{2,1}\|g\|_{2,1}\|h\|_{2,1},
$$

assuming $f, g$ and $h$ to be characteristic functions of finite measurable sets. By using the decomposition for $G$ described in Theorem 3.5, we know that the previous integral is equal to

$$
\int_{N} \int_{N} \sum_{j \in \mathbf{Z}} \sum_{j^{\prime} \in \mathbf{Z}} \int_{K} \int_{K} f\left(n \tau^{j} k\right) g\left(k^{-1} \tau^{-j} n^{-1} n^{\prime} \tau^{j^{\prime}} k^{\prime}\right) h\left(n^{\prime} \tau^{j^{\prime}} k^{\prime}\right) q^{-j-j^{\prime}} d n d n^{\prime} d k d k^{\prime} .
$$

Fubini's Theorem ensures that it is possible to change the order of integration because of our assumption on $f, g$ and $h$.

LEMmA 6.1. Let $f, g$ and $h$ be characteristic function of finite sets. Then

$$
\begin{aligned}
\int_{N} \int_{N} f\left(n \tau^{j} k\right) g\left(k^{-1} \tau^{-j} n^{-1} n^{\prime} \tau^{j^{\prime}} k^{\prime}\right) h\left(n^{\prime} \tau^{j^{\prime}} k^{\prime}\right) d n d n^{\prime} & \\
& \leqslant \min \left[f_{N}(k, j), h_{N}\left(k^{\prime}, j^{\prime}\right)\right] \int_{N} g\left(k^{-1} n \tau^{j} k^{\prime}\right) d n
\end{aligned}
$$

where $f_{N}(k, j)=\int_{N} f\left(n \tau^{j} k\right) d n$ and $h_{N}\left(k^{\prime}, j^{\prime}\right)=\int_{N} h\left(n^{\prime} \tau^{j^{\prime}} k^{\prime}\right) d n^{\prime}$ 
Proof: This follows because $\|f\|_{\infty}$ and $\|g\|_{\infty}$ are less than or equal to one and $N$ is unimodular.

Observe that $\|f\|_{2,1}^{2}=\sum_{j \in \mathbf{Z}} q^{-j} \int_{K} f_{N}(k, j) d k$ and $\|h\|_{2,1}^{2}=\sum_{j^{\prime} \in \mathbf{Z}} q^{-j^{\prime}} \int_{K} h_{N}\left(k^{\prime}, j^{\prime}\right) d k^{\prime}$. The main problem is to relate the expression containing $g$ with its $L^{2,1}$ norm. Ionescu's idea of using the link between the Cartan and Iwasawa decompositions in the semisimple Lie group case works in the tree context too. To find the link, we use the subset $T_{r, j}$ defined in formula (3.7). In view of Lemma 3.8 ,

$\int_{N} g\left(k^{-1} \tau^{-j} n \tau^{j^{\prime}} k^{\prime}\right) d n=q^{j} \int_{N} g\left(k^{-1} n_{1} \tau^{j^{\prime}-j} k^{\prime}\right) d n_{1}=q^{j} \sum_{r \geqslant\left|j^{\prime}-j\right|} \int_{T_{r, j^{\prime}-j}} g\left(k^{-1} n_{1} \tau^{j^{\prime}-j} k^{\prime}\right) d n_{1}$.

Recall that $\psi(r, j)=q^{-j / 2}\left|T_{r, j}\right|$ so, setting

$$
g^{N}\left(k, k^{\prime}, r, j\right)= \begin{cases}\frac{1}{\left|T_{r, j}\right|} \int_{T_{r, j}} g\left(k^{-1} n \tau^{j} k^{\prime}\right) d n & r \geqslant|j| \\ 0 & r<|j|\end{cases}
$$

we have

$$
\int_{N} g\left(k^{-1} \tau^{-j} n \tau^{j^{\prime}} k^{\prime}\right) d n=q^{\left(j^{\prime}+j\right) / 2} \sum_{r \in \mathbb{N}} g^{N}\left(k, k^{\prime}, r, j^{\prime}-j\right) \psi\left(r, j^{\prime}-j\right)
$$

and (6.12) may be dominated by

$$
\int_{K} \int_{K} \sum_{j, j^{\prime} \in \mathbf{Z}} \sum_{\tau \in \mathbb{N}} \min \left[f_{N}(k), h_{N}\left(k^{\prime}\right)\right] q^{-\left(j^{\prime}+j\right) / 2} g^{N}\left(k, k^{\prime}, r, j^{\prime}-j\right) \psi\left(r, j^{\prime}-j\right) d k d k^{\prime}
$$

The proposition below summarises how the link between the decompositions for $G$ allows us to relate the expression containing $g$ with its $L^{2,1}$ norm.

PROPOSITION 6.2. Let $g$ be a characteristic function of a finite set, denote by ${ }^{*} g^{*}(\cdot, \cdot, r)$ the double rearrangement of the function $\left(k, k^{\prime}\right) \mapsto g\left(k^{-1} \tau^{r} k^{\prime}\right)$ and let $g^{N}$ be as in formula (6.13). Then

(i) $\sum_{r \in \mathbb{N}} \int_{0}^{1} \int_{0}^{1}{ }^{*} g^{*}(s, t, r) q^{r} d s d t \leqslant\|g\|_{L^{2, i}(G)}^{2}$

$$
\leqslant((q+1) / q) \sum_{r \in \mathbf{N}} \int_{0}^{1} \int_{0}^{1}{ }^{*} g^{*}(s, t, r) q^{r} d s d t
$$

(ii) Let $D$ and $E$ be measurable subsets of $K$ of measure $\delta$ and $\varepsilon$. Then

$$
\int_{D} \int_{E} g^{N}\left(k, k^{\prime}, r, j\right) d k d k^{\prime} \leqslant \int_{0}^{\delta} \int_{0}^{\varepsilon}{ }^{*} g^{*}(s, t, r) d s d t
$$

Proof: Recall that, by the Cartan decomposition for $G$, for every $z \in G$ there exist $k_{1}(z), k_{2}(z)$ in $K$ and a unique $r$ in $\mathbb{N}$ such that $z=k_{1}(z)^{-1} \tau^{r} k_{2}(z)$. To prove part (i), first note that

$$
|\{z \in G:|z \cdot o|=r\}|=|\{x \in \mathfrak{X}: d(x, o)=r\}|=C(q, r) q^{r},
$$


where $C(q, 1)=1$ and $C(q, r)=(q+1) / q$ if $r \neq 1$. Fubini's Theorem and the left invariance of the normalised Haar measure $d k$ on $K$ imply that

$$
\begin{aligned}
\|g\|_{L^{2,1}(G)}^{2}=\int_{G} g(z) d z & =\int_{K} \int_{K} \int_{G} g\left(k^{-1} z k^{\prime}\right) d k d k^{\prime} d z \\
& =\int_{K} \int_{K} \sum_{r \in \mathbb{N}} \int_{\{z \in G:|z \cdot \cdot|=r\}} g\left(k^{-1} z k^{\prime}\right) d k d k^{\prime} d z \\
& =\int_{K} \int_{K} \sum_{r \in \mathbb{N}} \int_{\{z \in G:|z \cdot \cdot|=r\}} g\left(\left(k_{1} k\right)^{-1} \tau^{r} k_{2} k^{\prime}\right) d k d k^{\prime} d z \\
& =\sum_{r \in \mathbb{N}} C(q, r) \int_{K} \int_{K} g\left(k^{-1} \tau^{r} k^{\prime}\right) q^{r} d k d k^{\prime} \\
& =\sum_{r \in \mathbb{N}} C(q, r) \int_{0}^{1} \int_{0}^{1} g^{*}(s, t, r) q^{r} d s d t,
\end{aligned}
$$

by the property of rearrangements summarised in Lemma 2.1; this proves part (i). Note that part (ii) is trivial whenever $r<|j|$. If $r \geqslant|j|$, then the same argument used in part (i) and Lemma 3.8 show that

$$
\begin{aligned}
\int_{D} \int_{E} g^{N}\left(k, k^{\prime}, r, j\right) d k d k^{\prime} & =\int_{D} \int_{E} \frac{1}{\left|T_{r, j}\right|} \int_{T_{r, j}} g\left(k^{-1} n \tau^{j} k^{\prime}\right) d n d k d k^{\prime} \\
& =\int_{D} \int_{E} \frac{1}{\left|T_{r, j}\right|} \int_{T_{r, j}} g\left(\left(k_{1}(n) k\right)^{-1} \tau^{r} k_{2}(n) k^{\prime}\right) d n d k d k^{\prime} \\
& =\frac{1}{\left|T_{r, j}\right|} \int_{T_{r, j}} d n \int_{k_{1}(n) D} \int_{k_{2}(n) E} g\left(k^{-1} \tau^{r} k^{\prime}\right) d k d k^{\prime} \\
& \leqslant \int_{0}^{\delta} \int_{0}^{\varepsilon}{ }^{*} g^{*}(s, t, r) d s d t
\end{aligned}
$$

and the proposition follows.

Let $\tilde{f}(k)=\left(\sum_{j \in \mathbf{Z}} f_{N}(k, j) q^{-j}\right)^{1 / 2}$ and $\tilde{h}\left(k^{\prime}\right)=\left(\sum_{j^{\prime} \in \mathbf{Z}} h_{N}\left(k^{\prime}, j^{\prime}\right) q^{-j^{\prime}}\right)^{1 / 2}$.

LEMMa 6.3. Let $\tilde{f}^{*}$ and $\widetilde{h}^{*}$ be the rearrangement functions of $\tilde{f}$ and $\widetilde{h}$ and let ${ }^{*} g$ be as in Proposition 6.2. Then

$$
\int_{G} \int_{G} f(z) g\left(z^{-1} z^{\prime}\right) h\left(z^{\prime}\right) d z d z^{\prime} \leqslant C \int_{0}^{1} \int_{0}^{1} \sum_{r \in \mathbb{N}} \tilde{f}^{*}(s) \tilde{h}^{*}(t)^{*} g^{*}(s, t, r) q^{r / 2} d s d t .
$$

Proof: We know (see (6.14)) that $\int_{G} \int_{G} f(z) g\left(z^{-1} z^{\prime}\right) h\left(z^{\prime}\right) d z d z^{\prime}$ is dominated by

$$
\int_{K} \int_{K} \sum_{j, j^{\prime} \in \mathbb{Z}} \sum_{r \in \mathbb{N}} \min \left[f_{N}(k), h_{N}\left(k^{\prime}\right)\right] q^{-\left(j^{\prime}+j\right) / 2} g^{N}\left(k, k^{\prime}, r, j^{\prime}-j\right) \psi\left(r, j^{\prime}-j\right) d k d k^{\prime} .
$$


Let $\chi_{1}$ be the characteristic function of $\left\{\left(k, k^{\prime}, j, j^{\prime}\right): f_{N}(k, j) \leqslant h_{N}\left(k^{\prime}, j^{\prime}\right)\right\}$ and let $\chi_{2}$ be $1-\chi_{1}$. It is clear that

$$
f_{N}(k, j) \chi_{1}\left(k, k^{\prime}, j, j^{\prime}\right) \leqslant h_{N}\left(k^{\prime}, j^{\prime}\right) \text { and } h_{N}\left(k^{\prime}, j^{\prime}\right) \chi_{2}\left(k, k^{\prime}, j, j^{\prime}\right) \leqslant f_{N}(k, j),
$$

so that $\int_{G} \int_{G} f(z) g\left(z^{-1} z^{\prime}\right) h\left(z^{\prime}\right) d z d z^{\prime}$ may be dominated by the sum of two expressions like

$$
\begin{gathered}
\int_{K} \int_{K} \sum_{j, j^{\prime} \in \mathbf{Z}} \sum_{r \in \mathbf{N}} f_{N}(k, j) \chi_{1}\left(k, k^{\prime}, j, j^{\prime}\right) q^{-\left(j^{\prime}+j\right) / 2} g^{N}\left(k, k^{\prime}, r, j^{\prime}-j\right) \psi\left(r, j^{\prime}-j\right) d k d k^{\prime} \\
=\int_{K} \int_{K} \sum_{j, l \in \mathbf{Z}} \sum_{r \in \mathbf{N}} f_{N}(k, j) \chi_{1}\left(k, k^{\prime}, j, l+j\right) q^{-j-l / 2} g^{N}\left(k, k^{\prime}, r, l\right) \psi(r, l) d k d k^{\prime}
\end{gathered}
$$

Recall that $\tilde{f}(k)=\left(\sum_{j \in \mathbf{Z}} f_{N}(k, j) q^{-j}\right)^{1 / 2}$ and $\tilde{h}\left(k^{\prime}\right)=\left(\sum_{j^{\prime} \in \mathbf{Z}} h_{N}\left(k^{\prime}, j^{\prime}\right) q^{-j^{\prime}}\right)^{1 / 2}$ and set

$$
A\left(k, k^{\prime}, l\right)=\sum_{j \in \mathbf{Z}} f_{N}(k, j) \chi_{1}\left(k, k^{\prime}, j, j+l\right) q^{-j}
$$

so that expression (6.15) becomes

$$
\int_{K} \int_{K} \sum_{l \in \mathbf{Z}} \sum_{r \in \mathbf{N}} A\left(k, k^{\prime}, l\right) q^{-l / 2} g^{N}\left(k, k^{\prime}, r, l\right) \psi(r, l) d k d k^{\prime} .
$$

Since $A\left(k, k^{\prime}, l\right) \leqslant \widetilde{f}(k)^{2}$ and also

$$
A\left(k, k^{\prime}, l\right) \leqslant \sum_{j \in \mathbf{Z}} f_{N}(k, j) \chi_{1}\left(k, k^{\prime}, j, j+l\right) q^{-j} \leqslant \sum_{j \in \mathbf{Z}} h_{N}\left(k^{\prime}, j+l\right) q^{-(j+l-l)} \leqslant \widetilde{h}\left(k^{\prime}\right)^{2} q^{l},
$$

the expression (6.16) may be dominated by

$$
\begin{aligned}
\int_{K} \int_{K} \sum_{l \in I\left(k, k^{\prime}\right)+} \sum_{r \in \mathbf{N}} \tilde{f}(k)^{2} g^{N}\left(k, k^{\prime}, r, l\right) \psi(r, l) q^{-l / 2} d k d k^{\prime} \\
+\int_{K} \int_{K} \sum_{l \in I\left(k, k^{\prime}\right)-} \sum_{r \in \mathbf{N}} \widetilde{h}\left(k^{\prime}\right)^{2} g^{N}\left(k, k^{\prime}, r, l\right) \psi(r, l) q^{l / 2} d k d k^{\prime}
\end{aligned}
$$

where

$$
I\left(k, k^{\prime}\right)^{+}=\left\{l \in \mathbb{Z}: q^{-l / 2} \leqslant \widetilde{h}\left(k^{\prime}\right) / \widetilde{f}(k)\right\} \text { and } I\left(k, k^{\prime}\right)^{-}=\left\{l \in \mathbb{Z}: q^{-l / 2} \geqslant \widetilde{h}\left(k^{\prime}\right) / \widetilde{f}(k)\right\} .
$$

For any integers $n$ and $m$ define

$$
D_{m}=\left\{k \in K: \widetilde{f}(k) \in\left(q^{-(m+1) / 2}, q^{-m / 2}\right]\right\}
$$

and

$$
E_{n}=\left\{k^{\prime} \in K: \widetilde{h}\left(k^{\prime}\right) \in\left(q^{-(n+1) / 2}, q^{-n / 2}\right]\right\}
$$


Set

$$
D_{\infty}=\{k \in K: \tilde{f}(k)=0\} \text { and } E_{\infty}=\left\{k^{\prime} \in K: \tilde{h}\left(k^{\prime}\right)=0\right\}
$$

so that $K=\bigcup_{m} D_{m}=\bigcup_{n} E_{n}$. Let $\delta_{m}$ and $\varepsilon_{n}$ be the measures of the sets $D_{m}$ and $E_{n}$. Note that if $\left(k, k^{\prime}\right) \in D_{m} \times E_{n}$ then $I\left(k, k^{\prime}\right)^{+} \subseteq\{l \in \mathbb{Z}: l \geqslant n-m-1\}$, so that the first summand of (6.17) is dominated by

$$
\sum_{m, n \in \mathbf{Z}} \int_{D_{m}} \int_{E_{n}} \sum_{l \geqslant n-m-1} \sum_{r \in \mathbf{N}} q^{-m} g^{N}\left(k, k^{\prime}, r, l\right) \psi(r, l) q^{-l / 2} d k d k^{\prime}
$$

In view of Proposition 6.2, we see that $(6.18)$ is less than or equal to

$$
C \sum_{m, n \in \mathbf{Z}} \sum_{r \in \mathbf{N}} q^{-m}\left(\int_{0}^{\delta_{m}} \int_{0}^{\varepsilon_{n}} g^{*}(s, t, r) d s d t\right) \sum_{l \geqslant n-m-1} \psi(r, l) q^{-l / 2} .
$$

By using Lemma 3.11 we also know that $\psi(r, l) \leqslant q^{r / 2}$, then

$$
\sum_{l \geqslant n-m-1} \psi(r, l) q^{-l / 2} \leqslant C \sum_{l \geqslant n-m-1} q^{(r-l) / 2} \leqslant C q^{(-n+m+r) / 2},
$$

and $(6.19)$ is dominated by

$$
C \sum_{m, n \in \mathbf{Z}} \sum_{r \in \mathbf{N}} q^{-(m+n) / 2}\left(\int_{0}^{\delta_{m}} \int_{0}^{\varepsilon_{n}}{ }^{*} g^{*}(s, t, r) d s d t\right) q^{r / 2}
$$

Set

$$
S(s, t)=\sum_{m, n \in \mathbf{Z}}\left(q^{-(m+n) / 2} \chi_{\delta_{m}}(s) \chi_{\varepsilon_{n}}(t)\right)
$$

where $\chi_{\delta_{m}}=\chi_{\left(0, \delta_{m}\right)}$ and $\chi_{\varepsilon_{n}}=\chi_{\left(0, \varepsilon_{n}\right)}$. To conclude the estimate for the first term of (6.17), it is enough to show that

$$
S(s, t) \leqslant C \tilde{f}^{*}(s) \tilde{h}^{*}(t)
$$

Fix $s$ and $t$ in $(0,1)$. Observe that, since the measure of $K$ is finite, there are only a finitely many $m$ and $n$ such that $\delta_{m}>s$ and $\varepsilon_{n}>t$ respectively, so that $m_{s}=\min \{m \in$ $\left.\mathbb{Z}: \delta_{m}>s\right\}$ and $n_{t}=\min \left\{n \in \mathbb{Z}: \varepsilon_{n}>t\right\}$ are well defined. We have

$$
S(s, t) \leqslant \sum_{m>m_{s}} \sum_{n>n_{t}} q^{-(m+n) / 2} \leqslant C q^{-\left(m_{s}+n_{t}\right) / 2} .
$$

On the other hand if $f_{s}(k)=q^{-\left(m_{s}+1\right) / 2} \chi_{D_{m_{s}}}(k)$, it is clear that $f_{s}^{*}=q^{-\left(m_{s}+1\right) / 2} \chi_{\delta_{m_{s}}}$, that $f_{s}(k) \leqslant \tilde{f}(k)$ and finally that $f_{s}^{*} \leqslant \tilde{f}^{*}$. In particular $\tilde{f}^{*}(s) \geqslant f_{s}^{*}(s)=C_{1} q^{-m_{s} / 2}$. In the same way one shows that $\tilde{h}^{*}(t) \geqslant C_{2} q^{-n_{t} / 2}$, so that $(6.20)$ is proved. The other term in (6.17) may be dominated in a similar way so that the lemma is proved.

We can finally prove inclusion (6.11). 
THEOREM 6.4. Let $G$ denote the group of isometries of a homogeneous tree $\mathfrak{X}$ of degree $q+1$. The following continuous inclusion holds:

$$
L^{2,1}(G) * L^{2,1}(G) \subseteq L^{2, \infty}(G)
$$

Proof: Lemma 6.3 above ensures that it is enough to show

$$
\int_{0}^{1} \int_{0}^{1} \sum_{r \in \mathbb{N}} \tilde{f}^{*}(s) \widetilde{h}^{*}(t)^{*} g^{*}(s, t, r) q^{-r / 2} d s d t \leqslant C\|f\|_{2,1}\|g\|_{2,1}\|h\|_{2,1} .
$$

To do this, let $D$ be a constant, $\mathcal{U}=\left\{(s, t, r): \tilde{f}^{*}(s) * \widetilde{h}^{*}(t) \leqslant D q^{r / 2}\right\}$ and $\mathcal{V}=\{(s, t, r)$ : $\left.\tilde{f}^{*}(s) * \widetilde{h}^{*}(t) \geqslant D q^{r / 2}\right\}$. By Proposition 6.2,

$$
\int_{\mathcal{U}} \tilde{f}^{*}(s) \widetilde{h}^{*}(t)^{*} g^{*}(s, t, r) q^{r / 2} d s d t \leqslant D \sum_{r \in \mathbf{Z}} \int_{0}^{1} \int_{0}^{1}{ }^{*} g^{*}(s, t, r) q^{r} d s d t \leqslant D\|g\|_{L^{2,1}(G)}^{2} ;
$$

on the other hand, since ${ }^{*} g^{*}(s, t, r) \leqslant 1$, Lemma 2.1 implies that

$$
\begin{aligned}
\int_{\mathcal{V}} \tilde{f}^{*}(s) \widetilde{h}^{*}(t)^{*} g^{*}(s, t, r) q^{r / 2} d s d t & \leqslant \int_{0}^{1} \int_{0}^{1} \sum_{r:(s, t, r) \in \mathcal{V}} q^{r / 2} \widetilde{f}^{*}(s) \widetilde{h}^{*}(t) d s d t \\
& \leqslant C \int_{0}^{1} \int_{0}^{1} \frac{\left(\widetilde{f}(s) \widetilde{h}^{*}(t)\right)^{2}}{D} d s d t \\
& =C \int_{K} \int_{K} \frac{\tilde{f}(k)^{2} \widetilde{h}\left(k^{\prime}\right)^{2}}{D} d k d k^{\prime} \\
& =C \frac{\|f\|_{L^{2,1}(G)}^{2}\|h\|_{L^{2,1}(G)}^{2}}{D} .
\end{aligned}
$$

The last equality uses the fact that $f$ and $h$ are characteristic functions and then

$$
\begin{aligned}
\|f\|_{L^{2,1}(G)}^{2}=\int_{G} f(z) d z & =\int_{K} \sum_{\mathbf{Z}} \int_{N} f\left(n \tau^{j} k\right) q^{-j} d n d k \\
& =\int_{K} \sum_{\mathbf{Z}} f_{N}(k, j) q^{-j} d k=\int_{K} \tilde{f}(k)^{2} d k
\end{aligned}
$$

and $\|h\|_{L^{2,1}(G)}=\int_{K} \widetilde{h}\left(k^{\prime}\right)^{2} d k^{\prime}$. The theorem is proved by choosing $D$ equal to

$$
\left(\|g\|_{L^{2,1}(G)}\right)^{-1}\|f\|_{L^{2,1}(G)}\|h\|_{L^{2,1}(G)} .
$$

\section{ON RADIAL CONVOLUTORS OF $L^{p}(G)$}

In this section we illustrate a consequence of the Kunze-Stein phenomenon in the study of convolutors of $L^{p}(G)$. Recall that $(G, K)$ is a Gelfand pair, that is, $C_{c}(K \backslash G / K)$ is 
a commutative algebra with respect to convolution on $G$. In Theorem 7.1 below we summarise some properties of the spherical functions associated to $(G, K)$. Define the Laplace operator on $\mathfrak{X}$ by setting

$$
\mathcal{L} f(x)=f(x)-\frac{1}{q+1} \sum_{y: d(x, y)=1} f(y)
$$

and set

$$
\phi_{z}(x)= \begin{cases}\left(1+\frac{q-1}{q+1}|x|\right) q^{-|x| / 2} & \forall z \in \tau \mathbb{Z} \\ \left(1+\frac{q-1}{q+1}|x|\right) q^{-|x| / 2}(-1)^{|x|} & \forall z \in \tau / 2+\tau \mathbb{Z} \\ \mathbf{c}(z) q^{(i z-1 / 2)|x|}+\mathbf{c}(-z) q^{(-i z-1 / 2)|x|} & \forall z \in \mathbb{C} \backslash\{\tau \mathbb{Z} / 2\},\end{cases}
$$

where $\tau=2 \pi / \log q$ and $\mathbf{c}$ is the meromorphic function defined by

$$
\mathbf{c}(z)=\frac{q^{1 / 2}}{q+1} \frac{q^{1 / 2+i z}-q^{-1 / 2-i z}}{q^{i z}-q^{-i z}} \quad \forall z \in \mathbb{C} \backslash\{\tau \mathbb{Z} / 2\} .
$$

Theorem 7.1. Suppose $\mu \in \mathbb{C}$. Then there is a unique $K$-bi-invariant solution of the problem

$$
\left\{\begin{array}{l}
\mathcal{L} f=\mu f \\
f(o)=1
\end{array}\right.
$$

The spherical functions associated to $(G, K)$ are the solutions of (7.22) and are given by formula (7.21). The corresponding eigenvalue $\mu(z)$ is

$$
1-\frac{q^{1 / 2}}{q+1}\left(q^{i z}+q^{-i z}\right)
$$

The spherical functions $\phi_{i t}(x)$ are positive for all $t \in \mathbb{R}$, the map $t \mapsto \phi_{i t}(x)$ is increasing on $\mathbb{R}^{+}$and $\left|\phi_{s+i t}(x)\right| \leqslant \phi_{i t}(x)$ for all $x \in \mathfrak{X}$ and $s, t \in \mathbb{R}$.

For the proof, see [4, Chapter 2]. Observe that Theorem 6.4 and the bilinear interpolation theorem of Lions and Peetre [7] imply the following result (see also [3]).

TheOrem 7.2. Suppose that $G$ is the isometry group of $\mathfrak{X}$ and that $1<p<2$. Then the continuous inclusion

$$
L^{p, r}(G) * L^{p, s}(G) \subseteq L^{p, t}(G)
$$

holds if and only if $1 / t \leqslant 1 / r+1 / s-1$.

COROLLARY 7.3. If $1<p<2$, then $L^{p, 1}(G)$ is an algebra of convolutors for $L^{p}(G)$ and, in particular, $L^{p, 1}(K \backslash G / K)$ is a commutative algebra of convolutors. 
PROOF: It is enough to put first $r=s=t=1$ and then $r=1$ and $s=t=p$ in Theorem 7.2 above. Finally, $L^{p, 1}(K \backslash G / K)$ is commutative because $(G, K)$ is a Gelfand pair.

Before proceeding we need a theorem due to Pytlik [9].

THEOREM 7.4. A radial function $f$ on $\mathfrak{X}$ belongs to $L^{p, q}(K \backslash G / K)$ if and only if $r \mapsto q^{r / p} f(r)$ belongs to $L^{q}(\mathbb{N})$ and $\left\|q^{(\cdot) / p} f(\cdot)\right\|_{L^{q}(\mathbb{N})}$ is a norm equivalent to $\|\cdot\|_{L^{p, q}(K \backslash G / K)}$.

REMARK 7.5. Suppose $1<p<2$. Write $\delta(p)$ for $1 / p-1 / 2$ and let $S_{p}$ be the strip $\{z \in \mathbb{C}:|\operatorname{Im} z| \leqslant \delta(p)\}$. The explicit formula (7.21) show that a spherical function $\phi_{z}$ belongs to $L^{p^{\prime}, \infty}(K \backslash G / K)$ if and only if $z \in S_{p}$.

Herz's principe de majoration ensures that a positive $K$-bi-invariant function $f$ convolves $L^{p}(G)$ into itself if and only $\int_{G} f(z) \phi_{i \delta(p)}(z) d z$ is finite, where the spherical function $\phi_{i \delta(p)}$ is given by formula (7.21). This function is positive on $G$ and it is therefore natural to study the set of those $K$-bi-invariant functions $f$ such that $\|f\|_{C_{p}}$ is finite, where

$$
\|f\|_{C_{p}}=\int_{G}|f(z)| \phi_{i \delta(p)}(z) d z .
$$

By Pytlik's theorem this set of functions is equal to $L^{p, 1}(K \backslash G / K)$ (note that $\phi_{i \delta(p)} \sim$ $\left.q^{-(\cdot) / p^{\prime}}\right)$ and the two norms $\|\cdot\|_{C_{p}}$ and $\|\cdot\|_{p, 1}$ are equivalent. We write $C_{p}$ for $L^{p, 1}(K \backslash G / K)$ with the norm $\|\cdot\|_{C_{p}}$. The dual space $C_{p}^{*}$ of $C_{p}$ is then isomorphic to $L^{p^{\prime}, \infty}(K \backslash G / K)$ and the map $I: L^{p^{\prime}, \infty}(K \backslash G / K) \mapsto C_{p}^{*}$ given by setting

$$
I(\phi)(f)=\int_{G} f(x) \phi(x) d x \quad \forall f \in L^{p, 1}(K \backslash G / K),
$$

realises this isomorphism. The advantage of using $C_{p}$ instead of $L^{p, 1}(K \backslash G / K)$ is that $C_{p}$ is a Banach algebra. Note that Theorem 7.2 ensures that there exists a constant $C$ such that $\|f * g\|_{p, 1} \leqslant C\|f\|_{p, 1}\|g\|_{p, 1}$. By replacing $\|\cdot\|_{p, 1}$ with $\|\cdot\|_{p, 1}=C\|\cdot\|_{p, 1}$ we obtain another norm which makes $L^{p, 1}(K \backslash G / K)$ a Banach algebra. We prefer to use $C_{p}$ to avoid confusion and because the norm is more explicit. We already know that $C_{p}$ is a commutative algebra; if $f$ and $g$ are in $C_{c}(K \backslash G / K)$, then

$$
\begin{aligned}
\|f * g\|_{C_{p}} & =I\left(\phi_{i \delta(p)}\right)(|f * g|) \leqslant I\left(\phi_{i \delta(p)}\right)(|f| *|g|) \\
& =I\left(\phi_{i \delta(p)}\right)(|f|) I\left(\phi_{i \delta(p)}\right)(|g|)=\|f\|_{C_{p}}\|g\|_{C_{p}}
\end{aligned}
$$

so that $C_{p}$ is a unital commutative Banach algebra ( $\mathfrak{X}$ being a discrete space). We conclude by giving a description of its Gelfand spectrum $\Delta_{p}$, that is, the compact space of multiplicative functionals on $C_{p}$.

LEMMA 7.6. Suppose that $1<p<2$. A continuous linear functional $\Phi$ belongs to $\Delta_{p}$ if and only if there exists a spherical function $\phi \in L^{p^{\prime}, \infty}(K \backslash G / K)$ such that $\Phi=I(\phi)$. 
Proof: Let $\Phi \in \Delta_{p}$. Since $\Phi$ is continuous on $C_{p}$ there exists $\phi \in L^{p^{\prime}, \infty}(K \backslash G / K)$ such that $\Phi=I(\phi)$. Moreover $\Phi$ is multiplicative so that

$$
\int_{G}(f * g)(x) \phi(x) d x=\Phi(f * g)=\Phi(f) \Phi(g)=\int_{G} f(x) \phi(x) d x \int_{G} g(x) \phi(x) d x
$$

for all $f, g \in C_{c}(K \backslash G / K)$, that is, $\phi$ is spherical. On the other hand, assume $\phi \in$ $L^{p^{\prime}, \infty}(K \backslash G / K)$ is a spherical function. Since $I(\phi)$ is continuous and $C_{c}(K \backslash G / K)$ is dense in $L^{p, 1}(K \backslash G / K)$, the relations (7.25), true on $C_{c}(K \backslash G / K)$ by hypothesis, extend to all $C_{p}$. In other words $I(\phi)$ belongs to $\Delta_{p}$ and the lemma follows.

Lemma 7.6 above and Theorem 7.1 ensure that the map $\Lambda: \mu\left(S_{p}\right) \rightarrow \Delta_{p}$, defined by setting $\Lambda(w)=I\left(\phi_{z}\right)$ if $\mu(z)=w$, is well defined and is a bijection. We prove now that $\Lambda$ is actually an homeomorphism.

THEOREM 7.7. $\Lambda$ is a homeomorphism between $\mu\left(S_{p}\right)$ and $\Delta_{p}$.

Proof: We first show that the map $\lambda: z \mapsto I\left(\phi_{z}\right)$ is continuous. Consider the open set $\mathcal{U}$ in $\Delta_{p}$ given by

$$
\mathcal{U}=\mathcal{U}(f, \psi, \varepsilon)=\left\{\Phi \in \Delta_{p}:|(\Phi-\Psi)(f)|<\varepsilon\right\},
$$

where $f \in L^{p, 1}(K \backslash G / K), \varepsilon>0$ and $\Psi \in \Delta_{p}$ are fixed. To show that $\lambda^{-1}(\mathcal{U})$ is open in $S_{p} \subset \mathbb{C}$, consider $z_{0} \in \Lambda^{-1}(\mathcal{U})$. We have

$$
\begin{aligned}
|(\Lambda(z)-\Psi)(f)| & \leqslant\left|\left(\Lambda(z)-\Lambda\left(z_{0}\right)\right)(f)\right|+\left|\left(\Lambda\left(z_{0}\right)-\Psi\right)(f)\right| \\
& \leqslant \int_{G}\left|\phi_{z}(x)-\phi_{z_{0}}(x)\right||f(x)| d x+\eta,
\end{aligned}
$$

where $\eta=\left|\left(\Lambda\left(z_{0}\right)-\Psi\right)(f)\right|<\varepsilon$. In view of Theorem 7.1,

$$
\left|\phi_{z}(x)-\phi_{z_{0}}(x)\right| \leqslant\left|\phi_{z}(x)\right|+\left|\phi_{z_{0}}(x)\right| \leqslant 2 \phi_{i \delta(p)} \in L^{p^{\prime}, \infty}(K \backslash G / K),
$$

for all $z \in S_{p}$. It follows, by Lebesgue's Dominated Convergence Theorem, that if $\left|z-z_{0}\right|$ is small enough and $z \in S_{p}$, then $z \in \lambda^{-1}(\mathcal{U})$. This implies that $\lambda^{-1}(\mathcal{U})$ is open and $\lambda$ is continuous. Since $\mu$ is holomorphic, hence an open map, and $\Lambda^{-1}(\mathcal{U})=\mu\left(\lambda^{-1}(\mathcal{U})\right)$, this implies that $\Lambda$ is continuous too. Then $\Lambda$ is a continuous bijection of compact Hausdorff spaces, so a homeomorphism, and the theorem is proved.

\section{REFERENCES}

[1] M. Cowling, 'The Kunze-Stein phenomenon', Ann. Math. 107 (1978), 209-234.

[2] M. Cowling, 'Herz's "principe de majoration" and the Kunze-Stein phenomenon', in Harmonic analysis and number theory (Montreal, PQ, 1996) (Amer. Math. Soc., Providence, RI, 1997), pp. 73-88. 
[3] M. Cowling, S. Meda and A.G. Setti, 'An overview of harmonic analysis on the group of isometries of a homogeneous tree', Exposition. Math. 16 (1998), 385-423.

[4] A. Figà-Talamanca and C. Nebbia, Harmonic analysis and representation theory for groups acting on homogeneous trees (Cambridge University Press, Cambridge, 1991).

[5] A.D. Ionescu, 'An endpoint estimate for the Kunze-Stein phenomenon and related maximal operators', Ann. of Math. 152 (2000), 259-275.

[6] R.A. Kunze and E.M. Stein, 'Uniformly bounded representations and harmonic analysis of the $2 \times 2$ real unimodular group', Amer. J. Math. 82 (1960), 1-62.

[7] J.-L. Lions and J. Peetre, 'Sur une classe d'espaces d'interpolation', Inst. Hautes Études Sci. Publ. Math. 19 (1964), 5-68.

[8] N. Lohoué, 'Estimations $L^{p}$ des coefficients de représentation et opérateurs de convolution', Adv. in Math. 38 (1980), 178-221.

[9] T. Pytlik, 'Radial convolutors on free groups', Studia Math. 78 (1984), 179-183.

[10] E.M. Stein and G. Weiss, Introduction to Fourier analysis on Euclidean spaces, Princeton Mathematical Series 32 (Princeton University Press, Princeton, N.J., 1971).

Dipartimento di Matematica

Università di Milano

via Saldini 50

20133 Milano

Italia

e-mail: aveca@libero.it
School of Mathematics

UNSW

Sydney NSW 2052

Australia

e-mail: aveca@maths.unsw.edu.au 